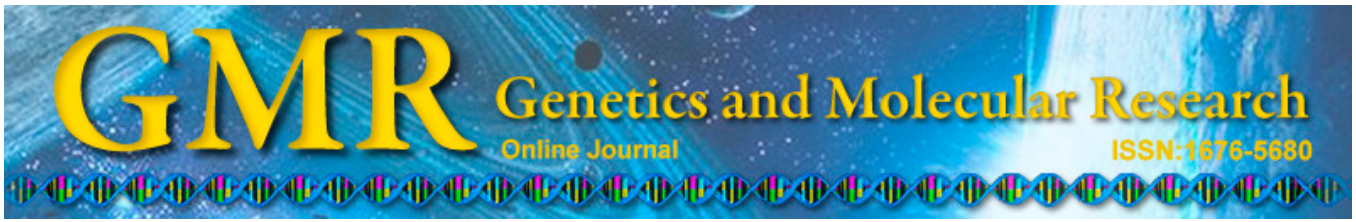

\title{
Clinical significance of lymphatic vessel invasion in stage I non-small cell lung cancer patients
}

\author{
K.F. Ma, X.Y. Chu and Y. Liu \\ Department of Thoracic Surgery, \\ General Hospital of the People's Liberation Army, Beijing, China \\ Corresponding author: X.Y. Chu \\ E-mail: xiangyangchu@163.com
}

Genet. Mol. Res. 14 (1): 1819-1827 (2015)

Received May 10, 2014

Accepted September 24, 2014

Published March 13, 2015

DOI http://dx.doi.org/10.4238/2015.March.13.10

\begin{abstract}
The aim of this retrospective study was to evaluate the prognostic influence of lymphatic vessel invasion (LVI) in stage I non-small cell lung cancer (NSCLC) patients. From January 2004 to December 2007, LVI was detected in 57 patients with T1N0M0 NSCLC; therefore, 114 patients with the same pathology, T stage, and surgery method, but without LVI, were selected as the control group to compare survival. The overall survival and relapse-free survival rates were estimated using the Kaplan-Meier method, log-rank test, and Cox proportional hazards analysis. The average follow-up length was 59.94 \pm 23.1 months. The 5-year overall survival rates of the LVI-negative and the LVI-positive groups were 90.54 and $70.1 \%$, respectively $(\mathrm{P}$ $=0.002$ ). A multivariate analysis revealed LVI to be an independent predictive factor (hazard ratio $=4.562 ; \mathrm{P}=0.004$ ). The 5-year overall survival rates for patients who received postoperative adjunctive therapy and those who did not in the LVI-positive group were 88.2 and $61.5 \%$, respectively, with a P value less than 0.05 in both univariate and multivariate analyses. LVI is a poor prognostic factor in stage I NSCLC patients; postoperative adjunctive therapy is needed to improve the
\end{abstract}


prognosis of NSCLC patients with LVI.

Key words: Lymphatic vessel invasion (LVI); Prognosis; Postoperative adjunctive therapy;

Stage I non-small cell lung cancer (NSCLC)

\section{INTRODUCTION}

Non-small cell lung cancer (NSCLC) constitutes approximately $80 \%$ of primary lung cancers, which is the most common malignancy worldwide (Jemal et al., 2011). Surgical resection remains the most effective therapy for NSCLC, especially early stage disease. However, the 5-year survival rate of patients with stage I NSCLC is only 79.4\%. Dozens of factors lead to disease recurrence and death for early stage NSCLC patients, and according to recent reports, angiogenesis and lymphangiogenesis are involved in tumor metastasis (Folkman, 1990; Padera et al., 2002). To investigate the clinical significance of lymphangiogenesis in NSCLC, we evaluated lymphatic vessel invasion (LVI). In the tumor-node-metastasis (TNM) staging system, LVI is not considered an independent prognostic factor. Thus, in accordance with lung cancer treatment guidelines, postoperative adjuvant therapy is not routinely recommended for stage I NSCLC, regardless of the presence of LVI (Scott et al., 2007).

We retrospectively reviewed the medical records of 57 NSCLC patients with LVI and 114 patients without LVI to investigate the relationship between LVI and prognosis and to determine whether postoperative adjuvant therapy should be administered to early stage NSCLC patients with LVI.

\section{MATERIAL AND METHODS}

\section{Patient selection}

We retrospectively reviewed the records of 171 patients with stage I NSCLC, admitted to our hospital from January 2004 to June 2007, who underwent surgery. This included 57 patients with LVI and 114 with the same pathology, T stage, and surgery method, but without LVI. All cases were classified into 2 subgroups according to the presence or absence of LVI (LVI+ or LVI-, respectively). Clinical data were obtained from medical records, and followup was conducted by direct patient contact; overall survival (OS), relapse-free survival (RFS), and metastasis location were recorded. The follow-up period ranged from 49 to 90 months, with an average follow-up of 59.94 months. Tumors were pathologically staged according to the seventh edition of the TNM classification for lung and pleural tumors of the International Union Against Cancer (Sobin et al., 2009). Histopathological studies were done based on World Health Organization criteria. Sections were stained with hematoxylin and eosin, and the Elastica van Gieson method was used to identify the existence of LVI. Patients' clinicopathologic characteristics are shown in Table 1.

\section{Statistical analyses}

OS and RFS were calculated using the Kaplan-Meier method, and differences in survival were tested by the log-rank method and Breslow method in univariate analyses. OS was 
from the date of pulmonary resection to the date of death from any cause. The last followup observation was censored when the patient was alive or lost to follow-up. To determine independent prognostic factors, multivariate analysis was conducted using the Cox proportional hazards model (Wald stepwise backward elimination method). P values less than 0.05 were considered to be statistically significant. All statistical analyses were performed with the PASW statistics software package version 13 (SPSS, Inc., Chicago, IL, USA).

\begin{tabular}{|c|c|c|c|}
\hline & LVI+ & LVI- & P value \\
\hline Cases $(\mathrm{N})$ & 57 & 114 & \\
\hline Age [years (mean)] & 57.29 & 53.08 & 0.841 \\
\hline Gender $(\mathrm{N})$ & & & 0.669 \\
\hline Male & 43 & 89 & \\
\hline Female & 14 & 25 & \\
\hline Surgical procedure $(\mathrm{N})$ & & & $\mathrm{S}$ \\
\hline Lobectomy & 48 & 96 & \\
\hline Limited resection & 9 & 18 & \\
\hline Tumor stage $(\mathrm{N})$ & & & $\mathrm{S}$ \\
\hline T1a & 32 & 64 & \\
\hline $\mathrm{T} 1 \mathrm{~b}$ & 25 & 50 & \\
\hline Histological type $(\mathrm{N})$ & & & $\mathrm{s}$ \\
\hline Adenocarcinoma & 33 & 66 & \\
\hline Squamous cell carcinoma & 14 & 28 & \\
\hline Others & 10 & 20 & \\
\hline Postoperative treatment $(\mathrm{N})$ & & & 0.205 \\
\hline Yes & 17 & 24 & \\
\hline No & 40 & 90 & \\
\hline
\end{tabular}

$\mathrm{P}$ values were analyzed using the chi-square test, and values $<0.05$ were considered to be significant. $\mathrm{LVI}+=$ lymphatic vessel invasion-positive; LVI- = lymphatic vessel invasion-negative; $\mathrm{S}=$ same proportion of each variable.

\section{RESULTS}

At the time of the last follow-up, 15 patients died in the LVI+ group, and 11 died in the LVI- group. The 5-year OS rates of the LVI+ and LVI- groups were 70.10 and $90.54 \%$, respectively. The survival rate curve is depicted in Figure 1. The difference in survival was statically significant $(\mathrm{P}=0.002)$. Patients' OS and RFS rate are shown in Table 2.

Metastasis and recurrence occurred in 19 patients in the LVI+ group and 16 patients in the LVI- group. The 5-year RFS rate in the LVI- group was $88.75 \%$, significantly higher than that in the LVI+ group $(69.68 \% ; \mathrm{P}=0.001)$. The survival rate curve is shown in Figure 2.

Patients' OS rates and RFS rates are shown in Table 2. A univariate analysis determined LVI (yes $v s$ no 70.1 vs $90.54 \% ; \mathrm{P}=0.002$ ) and postoperative adjuvant treatment (yes vs no 94.9 vs 79.7\%; $\mathrm{P}=0.024$ ) were significant poor prognostic factors. The remaining prognostic factors, such as T stage (T1a vs T1b: 85.71 vs $81.53 \%$; $\mathrm{P}=0.475$ ), surgical procedure (lobectomy vs limited resection: 84.72 vs $73.94 \%$; $\mathrm{P}=0.249$ ), and pathology (adenocarcinoma vs squamous carcinoma $v s$ others: 87.08 vs 81.57 vs $77.12 \% ; \mathrm{P}=0.398)$, were not significant predictive factors for survival (Table 3 ).

To determine whether these indicators were independently prognostic of OS, we also performed a multivariate analysis with a Cox proportional hazards model. The multivariate analysis revealed the independent prognostic influence of LVI and postoperative adjuvant therapy on OS, with hazard ratios of 4.562 (95\% confidence interval: $1.430-6.986 ; \mathrm{P}=0.004)$ and 3.161 (95\% confidence interval: 1.062-19.589; $\mathrm{P}=0.041)$, respectively (Table 4). 


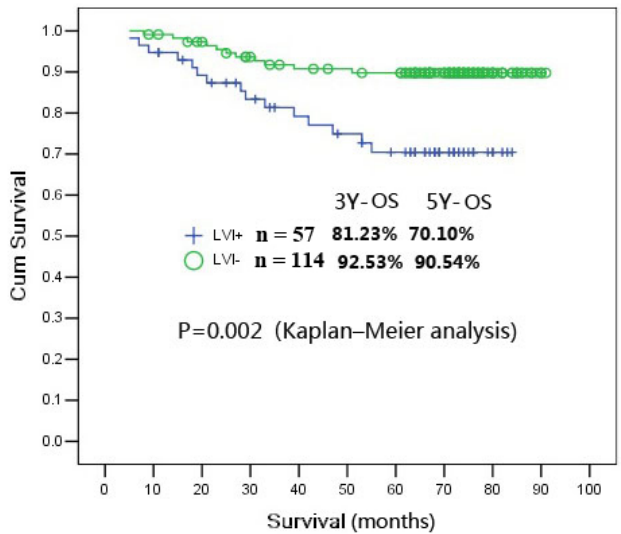

Figure 1. Overall survival of the LVI+ and LVI- groups (Kaplan-Meier method). The 5 -year overall survival (OS) rates of the lymphatic vessel invasion (LVI)-positive group and the LVI-negative group were 70.10 and $90.54 \%$, respectively, as determined by the Kaplan-Meier method and tested with the log-rank test. It was obvious that the prognosis of patients in the LVI-negative group was inferior to that of patients in the LVI-positive group.

Table 2. Overall survival and relapse-free survival rates in the LVI+ and LVI- groups.

\begin{tabular}{lccccc}
\hline & \multicolumn{2}{c}{ OS (\%) } & P value & \multicolumn{2}{c}{ RFS (\%) } \\
\cline { 2 - 3 } & LVI+ & LVI- & & LVI+ & LVI- \\
\hline 6 months & 98.25 & 100 & $\mathrm{P}=0.002$ & 98.25 & 100 \\
1 year & 94.67 & 99.12 & & 92.94 & 99.12 \\
2 years & 87.25 & 95.50 & & 82.01 & 97.31 \\
3 years & 81.23 & 92.53 & & 74.11 & 91.69 \\
5 years & 70.10 & 90.54 & 69.68 & 88.75 \\
\hline
\end{tabular}

P values were analyzed using the Kaplan-Meier method and tested using the log-rank test. P values $<0.05$ were considered to be significant. The survival differences for both OS and RFS were significant, with $\mathrm{P}$ value less than 0.05 . This indicates that LVI is a poor prognostic factor in stage I NSCLC patients. LVI+= lymphatic vessel invasion-positive; LVI- = lymphatic vessel invasion-negative; OS = overall survival; RFS = relapse-free survival; NSCLC = non-small cell lung cancer.

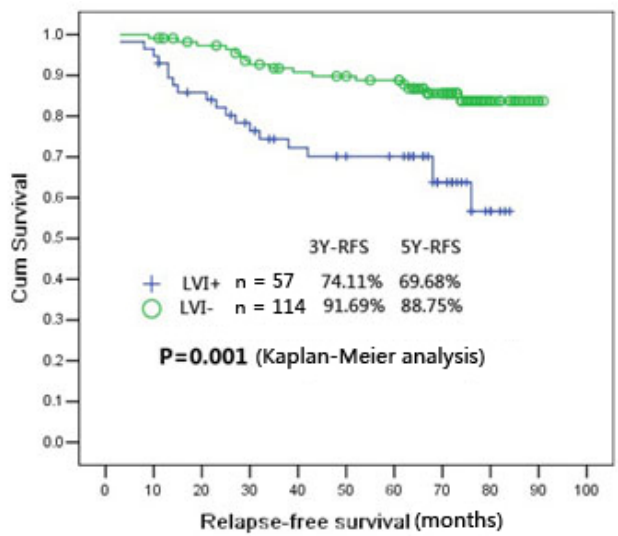

Figure 2. Relapse-free survival (RFS) of the LVI+ and LVI- groups (Kaplan-Meier method). The 5-year RFS rates of the lymphatic vessel invasion (LVI)-positive group and the LVI-negative group were 69.68 and $88.75 \%$, respectively. The survival difference was significant $(\mathrm{P}=0.001)$ by analysis using the Kaplan-Meier method and tested with the log-rank rest. 
Table 3. Relationship between categorical variables and patient survival by univariate prognostic analysis (Kaplan-Meier method).

\begin{tabular}{|c|c|c|c|}
\hline & Cases $(\mathrm{N})$ & 5-year survival (\%) & $\mathrm{P}$ value \\
\hline Pathology & 171 & & 0.398 \\
\hline Adenocarcinoma & 99 & 87.08 & \\
\hline Squamous carcinoma & 42 & 81.57 & \\
\hline Other & 30 & 77.12 & \\
\hline T stage & 171 & & \\
\hline T1a & 96 & 85.71 & 0.475 \\
\hline $\mathrm{T} 1 \mathrm{~b}$ & 75 & 81.53 & \\
\hline Postoperativetreatment & 171 & & \\
\hline No & 130 & 79.70 & 0.024 \\
\hline Yes & 41 & 94.90 & \\
\hline Surgery method & 171 & & \\
\hline Lobectomy & 144 & 84.72 & 0.249 \\
\hline Limited resection & 27 & 73.94 & \\
\hline LVI & 171 & & \\
\hline Yes & 57 & 70.10 & 0.002 \\
\hline No & 114 & 90.54 & \\
\hline
\end{tabular}

$\mathrm{P}$ values were analyzed using the Kaplan-Meier method and tested using the log-rank test. $\mathrm{P}$ values $<0.05$ were considered to be significant. Postoperative treatment, postoperative adjuvant chemotherapy and radiotherapy; $\mathrm{T}$ stage, T1a and T1b according to the TNM classification system of the International Union Against Cancer (7th edition); LVI = lymphatic vessel invasion.

Table 4. Multivariate analysis of risk factors for overall survival in stage I NSCLC patients using the Cox proportional hazards model.

\begin{tabular}{lccrr}
\hline & P value (Wald) & \multicolumn{2}{c}{ 95\%CI for EXP B } \\
\cline { 3 - 5 } & & EXP (B) & Min & Max \\
\hline Pathology & 0.257 & 1.356 & 0.800 & 2.298 \\
LVI & 0.004 & 4.562 & 1.430 & 6.986 \\
T stage & 0.476 & 0.746 & 0.333 & 1.671 \\
Postoperative treatment & 0.041 & 3.161 & 1.062 & 19.589 \\
Surgery method & 0.658 & 1.264 & 0.449 & 3.556 \\
\hline
\end{tabular}

P values $<0.05$ were considered to be statistically significant. $\mathrm{CI}=$ confidence interval; Pathology $=$ adenocarcinoma, squamous carcinoma, and other NSCLC types; LVI = lymphatic vessel invasion; $\mathrm{T}$ stage $=\mathrm{T} 1 \mathrm{a}$ and $\mathrm{T} 1 \mathrm{~b}$ according to the TNM classification system of the International Union Against Cancer (7th edition); Postoperative treatment = postoperative adjuvant chemotherapy and radiotherapy; Surgery method = lobectomy and limited resection.

There were 17 patients who received postoperative adjuvant therapy in the LVI+ group, and the 5-year survival rate was $88.2 \%$, significantly higher than that of remaining patients $(61.5 \% ; \mathrm{P}=0.047$; Figure 3). Metastasis locations are shown in Figure 4.

\section{DISCUSSION}

The TNM staging system is the most effective instrument for assessing NSCLC, and lymph node metastasis is a well-known poor prognosis factor. For stage I NSCLC patients, the 5 -year survival rate is $79 \%$, which means that up to $20 \%$ of patients will experience distant or local recurrence (Sawabata et al., 2010). This indicates that there are unknown poor prognostic factors in early stage NSCLC that result in unfortunate outcomes for patients. In recent years, more and more authors have emphasized the influence of LVI on the poor prognosis of NSCLC 


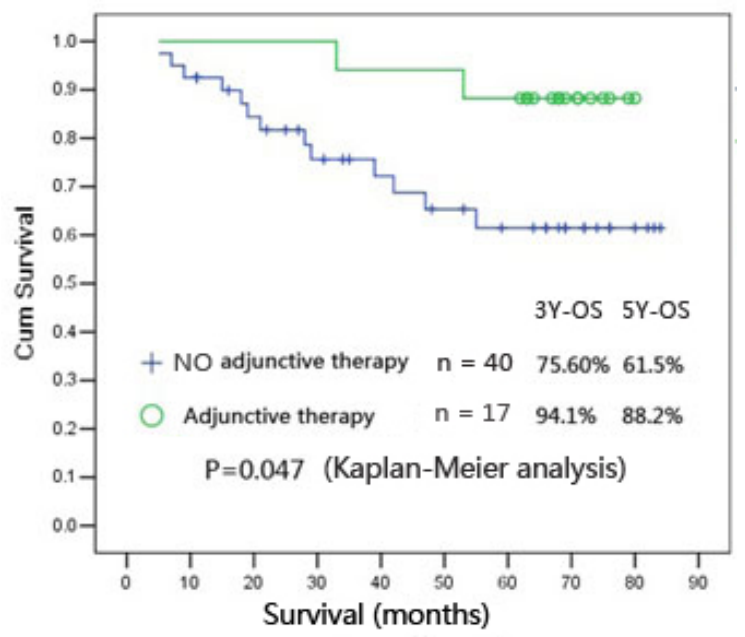

Figure 3. Overall survival (OS) of patients in the LVI+ group by receipt of postoperative adjunctive therapy (Kaplan-Meier method). In the lymphatic vessel invasion (LVI)-positive group, there were 17 patients who received adjunctive therapy, including chemotherapy and radiation therapy. The 5-year OS rate of the 17 patients was $88.2 \%$, significantly better than that of the remaining 40 patients who did not receive adjunctive therapy (5-year survival $61.5 \% ; \mathrm{P}=0.047)$.

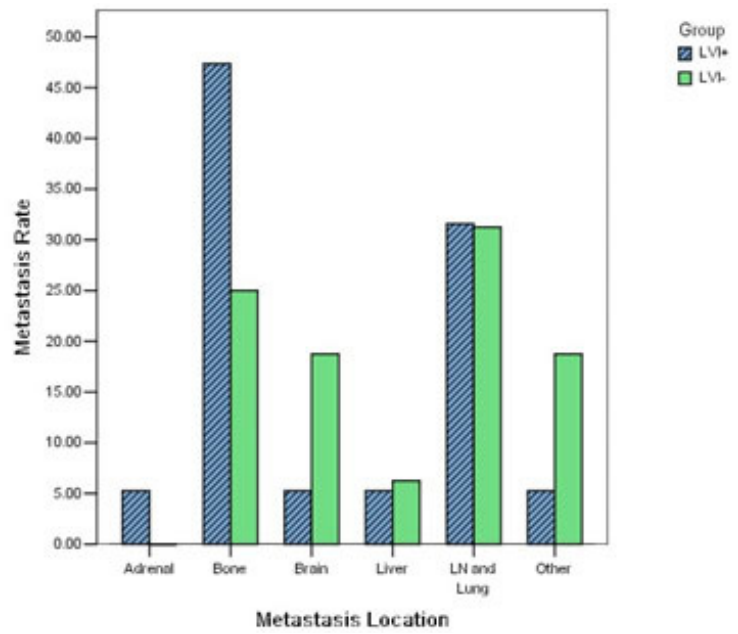

Figure 4. Metastasis location of two groups. Metastasis and disease recurrence occurred in 19 patients in the lymphatic vessel invasion (LVI)-positive group and 16 patients in the LVI-negative group. In the LVI-positive group, bone metastasis occurred in 9 patients, lymph node (LN) and lung metastasis occurred in 7 patients, and liver, brain, and adrenal metastasis each occurred in 1 patient. However, in the LVI-negative group, LN and lung metastasis occurred in 6 patients, bone metastasis occurred in 4 patients, brain metastasis occurred in 3 patients, liver metastasis occurred in 1 patient, and there was no case of adrenal metastasis.

patients, including stage I patients, who have undergone radical resection (Ichinose et al., 1994; Kessler et al., 1996; Duarte et al., 1998; Port et al., 2003; Gabor et al., 2004; Sakao et al., 2004); the frequency of radical resection generally ranges from 15 to $36 \%$ in the literature (Rigau et 
al., 2002; Funai et al., 2011). In our research, we retrospectively studied the follow-up records of stage I NSCLC patients to analyze the relationship between LVI and prognosis.

The OS and RFS rates in the LVI+ group were both inferior to those in the LVI- group by univariate analysis, and these differences were statistically significant. This demonstrates that LVI is a poor prognostic factor for OS and tumor metastasis. Tumor metastasis and progression are complicated and multistep processes that begin with local invasion of tumor cells into the host stroma within or surrounding the primary tumor. Tumor cells can detach and arrest in the microvasculature and then penetrate the peripheral lymphatic system (Hanahan and Weinberg, 2011). LVI indicates that the tumors are in a metastatic phase and also predicts a poor postoperative prognosis. Tumor microvessel formation is also a key factor of lung cancer metastasis and indicates poor prognosis from the molecular level (Angeletti et al., 1996; Pastorino et al., 1997; Offersen et al., 2001). Tumor cells may secrete and release a variety of active factors, such as vascular endothelial growth factor, to induce tumor angiogenesis (Mattern et al., 1996; Fontanini et al., 1997; O'Byrne et al., 2000), thus contributing to vascular invasion, including LVI and blood vascular invasion. Goldstein et al. (1999), who reported that LVI is the worst prognostic factor in stage I NSCLC, and Shoji et al. (2010), who reported that blood vascular invasion is the worst prognostic factor in stage I NSCLC, hold opposite views. The argument will not cease because survival results between patients with blood vessel invasion (BVI) and LVI have not been compared, because no patients with BVI have been recruited to survival studies. Cote et al. (1995) concluded that LVI plays a very important role in bone metastasis in NSCLC patients. In our research, there were 19 patients $(33.33 \%$; 19/57) who had distant metastasis or recurrence; $47.37 \%(9 / 19)$ of patients in the LVI+ group had bone metastasis as the most likely metastasis location, and only $16(14.04 \%, 16 / 114)$ patients in the LVI- group had metastasis, with $4(25.0 \%, 4 / 16)$ cases occurring in the bone. However, the difference in metastasis locations between the 2 groups was not statically significant, with a $\mathrm{P}$ value greater than 0.05 . LVI is also highly correlated with intrapulmonary metastasis in nonsmall cell cancer of the lung, and in some reports, it plays an important role in the metastasis and spread of lung satellite lesions (Fujisawa et al., 1995).

The survival of stage III NSCLC patients could clearly be prolonged through postoperative adjunctive therapy (Ettinger et al., 2010); it is unclear whether postoperative adjunctive therapy will also benefit stage I NSCLC patients with LVI. In studies by Kelsey et al. (2009) and Saynak et al. (2011), adjuvant chemotherapy and radiotherapy were ineffective for patients with LVI; however, Kato et al. (2004) and Tsuchiya et al. (2007) demonstrated the usefulness of oral uracil-tegafur chemotherapy after resection for stage I NSCLC. Our univariate analysis provides strong evidence that postoperative adjunctive therapy is a prognostic factor for survival in patients with NSCLC. In addition, in the multivariate analysis with the Cox regression model, postoperative treatment $(\mathrm{P}=0.041)$ was also identified as a significant independent predictor of OS and RFS. A total of 15 patients received postoperative adjunctive therapy in the LVI+ group, and among these patients, the 5 -year survival rate was $88.2 \%$, better than patients who did not receive postoperative adjunctive therapy, with a 5 -year survival rate of only $61.5 \%$. This suggests that adjuvant therapy should be a common treatment option for patients.

Most lung cancer treatment failures occur within the first 2 years after surgery (Poleri et al., 2003), and once metastasis occurs, the survival rate is generally less than 3 years (Kato et al., 2012). A total of $10(52.63 \% ; 10 / 19)$ patients suffered from tumor metastasis in the first 2 years after their operation in the LVI+ group, and in the LVI- group, tumor metastasis only occurred in $3(18.75 \% ; 3 / 16)$ patients; this difference was statically significant, with a $\mathrm{P}$ value 
less than 0.05 . Some authors believe that LVI is also a predictor of early mortality (Fujisawa et al., 1995; Pechet et al., 2004); 46.67\% (7/15) and 45.45\% (5/11) of the deaths in this study occurred in the first 2 years for the LVI+ group and LVI- group, respectively. However, this difference was not statistically significant $(\mathrm{P}>0.05)$.

Recently, D2-40, a new, selective, monoclonal immunohistochemical marker, has been demonstrated to be useful in the diagnosis of NSCLC LVI, and there have been few reports about the correlation of D2-40-positive LVI and prognosis (Kadota et al., 2010; Schuchert et al., 2011). The use of D2-40 for the detection of LVI has also been reported for cancers of the breast, stomach, colon, prostate, cervix, endometrium, and skin (melanoma, squamous cell carcinoma) (Kahn and Marks, 2002; Dumoff et al., 2005; Shida et al., 2005; Van der Auwera et al., 2005). It also offers a useful approach to identify aggressive lung squamous cell carcinoma, while others have argued the opposite (Faoro et al., 2008; Iwakiri et al., 2009).

In conclusion, the prognostic value of LVI is underestimated in stage I NSCLC, and a multicenter, randomized, prospective study is needed to confirm the findings. Our data demonstrated that postoperative adjuvant treatment in patients with LVI can improve prognosis. However, a prospective, randomized, controlled trial that evaluates the impact of adjuvant chemotherapy on survival in patients with stage I NSCLC and LVI is also needed.

\section{ACKNOWLEDGMENTS}

Thanks to Professor Chu XiangYang and Professor Xue Zhi Qiang for their invaluable assistance.

\section{REFERENCES}

Angeletti CA, Lucchi M, Fontanini G, Mussi A, et al. (1996). Prognostic significance of tumoral angiogenesis in completely resected late stage lung carcinoma (stage IIIA-N2). Impact of adjuvant therapies in a subset of patients at high risk of recurrence. Cancer 78: 409-415.

Cote RJ, Beattie EJ, Chaiwun B, Shi SR, et al. (1995). Detection of occult bone marrow micrometastases in patients with operable lung carcinoma. Ann. Surg. 222: 415-423.

Duarte IG, Bufkin BL, Pennington MF, Gal AA, et al. (1998). Angiogenesis as a predictor of survival after surgical resection for stage I non-small-cell lung cancer. J. Thorac. Cardiovasc. Surg. 115: 652-658.

Dumoff KL, Chu C, Xu X, Pasha T, et al. (2005). Low D2-40 immunoreactivity correlates with lymphatic invasion and nodal metastasis in early-stage squamous cell carcinoma of the uterine cervix. Mod. Pathol. 18: 97-104.

Ettinger DS, Akerley W, Bepler G, Blum MG, et al. (2010). Non-small cell lung cancer. J. Natl. Compr. Canc. Netw. 8: $740-801$.

Faoro L, Hutto JY, Salgia R, El-Zayaty SA, et al. (2008). Lymphatic vessel density is not associated with lymph node metastasis in non-small cell lung carcinoma. Arch. Pathol. Lab. Med. 132: 1882-1888.

Folkman J (1990). What is the evidence that tumors are angiogenesis dependent? J. Natl. Cancer Inst. 82: 4-6.

Fontanini G, Vignati S, Lucchi M, Mussi A, et al. (1997). Neoangiogenesis and p53 protein in lung cancer: their prognostic role and their relation with vascular endothelial growth factor (VEGF) expression. Br. J. Cancer 75: 1295-1301.

Fujisawa T, Yamaguchi Y, Saitoh Y, Hiroshima K, et al. (1995). Blood and lymphatic vessel invasion as prognostic factors for patients with primary resected nonsmall cell carcinoma of the lung with intrapulmonary metastases. Cancer 76: 2464-2470.

Funai K, Sugimura H, Morita T, Shundo Y, et al. (2011). Lymphatic vessel invasion is a significant prognostic indicator in stage IA lung adenocarcinoma. Ann. Surg. Oncol. 18: 2968-2972.

Gabor S, Renner H, Popper H, Anegg U, et al. (2004). Invasion of blood vessels as significant prognostic factor in radically resected T1-3N0M0 non-small-cell lung cancer. Eur. J. Cardiothorac. Surg. 25: 439-442.

Goldstein NS, Mani A, Chmielewski G, Welsh R, et al. (1999). Prognostic factors in T1 NO MO adenocarcinomas and bronchioloalveolar carcinomas of the lung. Am. J. Clin. Pathol. 112: 391-402.

Hanahan D and Weinberg RA (2011). Hallmarks of cancer: the next generation. Cell 144: 646-674.

Genetics and Molecular Research 14 (1): 1819-1827 (2015)

CFUNPEC-RP www.funpecrp.com.br 
Ichinose Y, Yano T, Yokoyama H, Inoue T, et al. (1994). The correlation between tumor size and lymphatic vessel invasion in resected peripheral stage I non-small-cell lung cancer. A potential risk of limited resection. J. Thorac. Cardiovasc. Surg. 108: 684-686.

Iwakiri S, Nagai S, Katakura H, Takenaka K, et al. (2009). D2-40-positive lymphatic vessel density is a poor prognostic factor in squamous cell carcinoma of the lung. Ann. Surg. Oncol. 16: 1678-1685.

Jemal A, Bray F, Center MM, Ferlay J, et al. (2011). Global cancer statistics. CA Cancer J. Clin. 61: 69-90.

Kadota K, Huang CL, Liu D, Nakashima N, et al. (2010). The clinical significance of the tumor cell D2-40 immunoreactivity in non-small cell lung cancer. Lung Cancer 70: 88-93.

Kahn HJ and Marks A (2002). A new monoclonal antibody, D2-40, for detection of lymphatic invasion in primary tumors. Lab. Invest. 82: 1255-1257.

Kato H, Ichinose Y, Ohta M, Hata E, et al. (2004). A randomized trial of adjuvant chemotherapy with uracil-tegafur for adenocarcinoma of the lung. N. Engl. J. Med. 350: 1713-1721.

Kato T, Ishikawa K, Aragaki M, Sato M, et al. (2012). Angiolymphatic invasion exerts a strong impact on surgical outcomes for stage I lung adenocarcinoma, but not non-adenocarcinoma. Lung Cancer 77: 394-400.

Kelsey CR, Marks LB, Hollis D, Hubbs JL, et al. (2009). Local recurrence after surgery for early stage lung cancer: an 11-year experience with 975 patients. Cancer 115: 5218-5227.

Kessler R, Gasser B, Massard G, Roeslin N, et al. (1996). Blood vessel invasion is a major prognostic factor in resected non-small cell lung cancer. Ann. Thorac. Surg. 62: 1489-1493.

Mattern J, Koomagi R and Volm M (1996). Association of vascular endothelial growth factor expression with intratumoral microvessel density and tumour cell proliferation in human epidermoid lung carcinoma. Br. J. Cancer 73: 931-934.

O'Byrne KJ, Koukourakis MI, Giatromanolaki A, Cox G, et al. (2000). Vascular endothelial growth factor, plateletderived endothelial cell growth factor and angiogenesis in non-small-cell lung cancer. Br. J. Cancer 82: 1427-1432.

Offersen BV, Pfeiffer P, Hamilton-Dutoit S and Overgaard J (2001). Patterns of angiogenesis in non-small cell lung carcinoma. Cancer 91: 1500-1509.

Padera TP, Kadambi A, di TE, Carreira CM, et al. (2002). Lymphatic metastasis in the absence of functional intratumor lymphatics. Science 296: 1883-1886.

Pastorino U, Andreola S, Tagliabue E, Pezzella F, et al. (1997). Immunocytochemical markers in stage I lung cancer: relevance to prognosis. J. Clin. Oncol. 15: 2858-2865.

Pechet TT, Carr SR, Collins JE, Cohn HE, et al. (2004). Arterial invasion predicts early mortality in stage I non-small cell lung cancer. Ann. Thorac. Surg. 78: 1748-1753.

Poleri C, Morero JL, Nieva B, Vázquez MF, et al. (2003). Risk of recurrence in patients with surgically resected stage I non-small cell lung carcinoma: histopathologic and immunohistochemical analysis. Chest 123: 1858-1867.

Port JL, Kent MS, Korst RJ, Libby D, et al. (2003). Tumor size predicts survival within stage IA non-small cell lung cancer. Chest 124: 1828-1833.

Rigau V, Molina TJ, Chaffaud C, Huchon G, et al. (2002). Blood vessel invasion in resected non small cell lung carcinomas is predictive of metastatic occurrence. Lung Cancer 38: 169-176.

Sakao Y, Nakazono T, Sakuragi T, Natsuaki M, et al. (2004). Predictive factors for survival in surgically resected clinical IA peripheral adenocarcinoma of the lung. Ann. Thorac. Surg. 77: 1157-1161.

Sawabata N, Asamura H, Goya T, Mori M, et al. (2010). Japanese Lung Cancer Registry Study: first prospective enrollment of a large number of surgical and nonsurgical cases in 2002. J. Thorac. Oncol. 5: 1369-1375.

Saynak M, Veeramachaneni NK, Hubbs JL, Nam J, et al. (2011). Local failure after complete resection of N0-1 non-small cell lung cancer. Lung Cancer 71: 156-165.

Schuchert MJ, Schumacher L, Kilic A, Close J, et al. (2011). Impact of angiolymphatic and pleural invasion on surgical outcomes for stage I non-small cell lung cancer. Ann. Thorac. Surg. 91: 1059-1065.

Scott WJ, Howington J, Feigenberg S, Movsas B, et al. (2007). Treatment of non-small cell lung cancer stage I and stage II: ACCP evidence-based clinical practice guidelines (2nd edition). Chest 132: 234S-242S.

Shida A, Fujioka S, Ishibashi Y, Kobayashi K, et al. (2005). Prognostic significance of vascular endothelial growth factor D in gastric carcinoma. World J. Surg. 29: 1600-1607.

Shoji F, Haro A, Yoshida T, Ito K, et al. (2010). Prognostic significance of intratumoral blood vessel invasion in pathologic stage IA non-small cell lung cancer. Ann. Thorac. Surg. 89: 864-869.

Sobin L, Gospodarowicz M and Wittekind C (2009). International Union Against Cancer (UICC) TNM Classification of Malignant Tumours. 7th edn. Wiley-Blackwell, Oxford.

Tsuchiya T, Akamine S, Muraoka M, Kamohara R, et al. (2007). Stage IA non-small cell lung cancer: vessel invasion is a poor prognostic factor and a new target of adjuvant chemotherapy. Lung Cancer 56: 341-348.

Van der Auwera I, Van den Eynden GG, Colpaert CG, Van Laere SJ, et al. (2005). Tumor lymphangiogenesis in inflammatory breast carcinoma: a histomorphometric study. Clin. Cancer Res. 11: 7637-7642. 